\title{
Non-Hertzian rolling contact stress analysis
}

\author{
C. H. Liu \& W.-E. Hsu \\ Department of Mechanical \& Electro-mechanical Engineering, \\ Tamkang University, Tamsui, Taipei Shien, Taiwan, 251
}

\begin{abstract}
In a previous study the three-dimensional rolling contact problem under Hertzian pressure has been dealt with. Two new initial guesses for the Newton-Raphson method were proposed, which always lead to convergent solutions for tangential stresses. However, non-Hertzian contact often appears in moderately used contact elements. The present study extends the previous study to cases with non-Hertzian contact. In particular the counter-formal case is treated. In this case the contact region can still be bounded by a plane, although the contact pressure is not Hertzian. The previous numerical algorithm is used to treat counter-formal contacts, and convergent results can always be obtained.
\end{abstract}

Keywords: rolling contact, rail and wheel contact, non-Hertzian contact, computational stress analysis.

\section{Introduction}

Rolling contact occurs in many mechanical pairs, such as gear and pinion, cam and follower, wheel and rail, and also in ball to ball contacts in bearings. Many analytical as well as numerical techniques have been suggested to solve for rolling contact stresses. Basically these techniques can be grouped into the following three categories: the integral equation method [1-5], the method based on variational principles [6,7], and the mixed method [8-10] that makes use of both of the two preceding methods. Among the various techniques based upon integral equations, the numerical procedure developed by Liu and Paul [5] may determine tangential contact stress distribution for elastically similar bodies in rolling contact. Their iterative procedure showed fast convergence for cases with small spins, but might fail to converge for cases with even moderate values of spin. In a previous study by Liu and Hsu [11], Liu and Paul's numerical scheme was improved by using two new initial guesses, so that it might also converge 
under very large spins. In Liu and Paul's original study, both Hertzian and nonHertzian contacts were treated, but only cases with Hertzian contact pressure were presented by Liu and Hsu. The purpose of this study, therefore, is to extend this previous study to cases with non-Hertzian contacts.

The Hertzian pressure distribution was obtained under the following assumptions: 1. the contact region is very small so that it can be bounded in a plane; 2 . in the vicinity of the contact region the two contacting bodies can be approximated by quadratic surfaces; and 3 . the surfaces in contact are frictionless. While these are valid assumptions for new contacting surfaces, for moderately used contact elements, however, the above assumptions are generally not valid, and the corresponding contact pressures are non-Hertzian. In wheel and rail contact, although the pressure is non-Hertzian, but the contact region can still be confined on a plane [12]. In this study we assume that the loading is monotonically increasing, and that a fixed ratio of normal to tangential forces is maintained, so that only the final force values are considered.

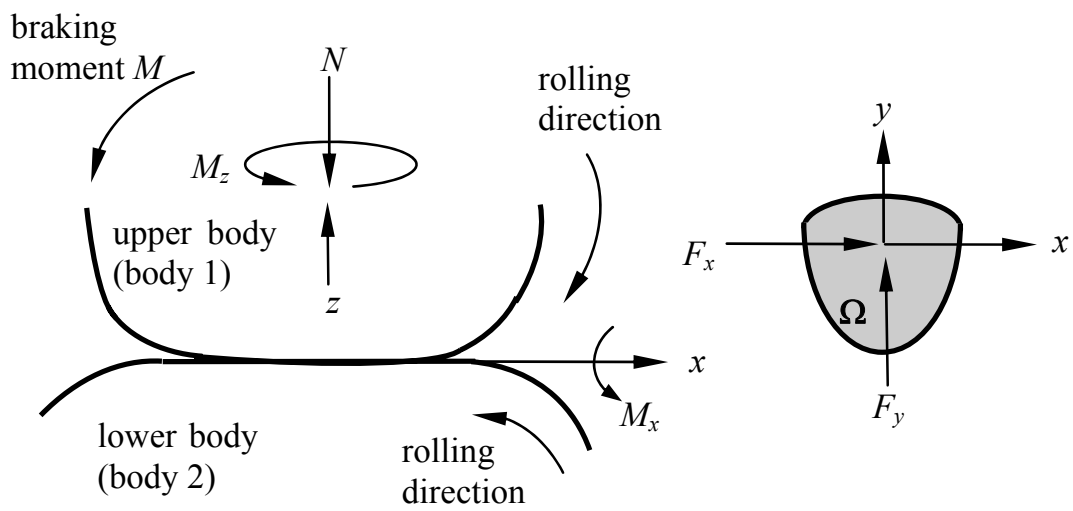

(a)

(b)

Figure 1: (a) two bodies in rolling contact; (b) tangential forces on contact region $\Omega$.

\section{Kinematic equation of rolling contact}

When two rolling bodies are pressed by a normal force $N$, the contact region $\Omega$ develops in the $x y$ plane, as Fig. 1 shows. In addition to the normal force $N$, tangential force $\left(F_{x}, F_{y}\right)$ and twisting moment $M_{z}$ are also transmitted in the contact region. If the two bodies in contact are elastically identical, namely, they have the same elastic properties, then the problem can be separated into two parts: the normal problem and the tangential problem [13]. In the normal problem, the contact region $\Omega$ and the pressure distribution $p(x, y)$ in the region are determined. Tangential tractions $T_{x}(x, y)$ and $T_{y}(x, y)$ in the contact region are then determined in the tangential problem. This separation is valid for elastically identical materials because the tangential tractions imposed later on the contact 
region do not affect the conformity of the two bodies in contact. Hence the normal pressure and the size of the contact region remain the same. In this study we assume the two bodies rolling over each other are elastically identical. The non-Hertzian contact region $\Omega$ and the pressure $p(x, y)$ in $\Omega$ are given. The purpose is to determine the tangential tractions $T_{x}(x, y)$ and $T_{y}(x, y)$ in the contact region $\Omega$.

When a braking moment $M$ in the direction of $-y$ is applied to body 1 , a tangential force $F_{x}$ develops in the contact region $\Omega$ to oppose the tendency of body 1 to slide over body 2. Likewise, a moment $M_{x}$ along the $x$ axis creates a tangential $F_{y}$ in $\Omega$. Figure 1(b) shows $F_{x}$ and $F_{y}$ in the contact region on body 2 . As a result of $F_{x}$ and $F_{y}$, certain points on the upper body (body 1) may slip over the contacting points on the lower body (body 2 ). The slippages $s_{x}$ and $s_{y}$ defined by

$$
\left(s_{x}, s_{y}\right)=\left(\mathrm{v}_{x 1}-\mathrm{v}_{x 2}, \mathrm{v}_{y 1}-\mathrm{v}_{y 2}\right) / V_{0}
$$

represent the velocities at a point $(x, y)$ on body 1 relative to the point occupying the same position but on body 2 . The normalization constant $V_{0}$ is the rolling velocity when the two rolling bodies are rigid, that is, when they do not deform. The contact region hence reduces to a single contact point. In the case of gear and pinion pairs, rolling velocity $V_{0}$ is the velocity of the contact point, and in the case of wheel-rail rolling contact, $V_{0}$ is the velocity of the center of the rigid wheel. Johnson [1,2] derived the following kinematic equation for a state of steady rolling

$$
\left(s_{x}, s_{y}\right)=\left(v_{x}, v_{y}\right)+\phi(-y, x)+2 \frac{\partial}{\partial x}\left(u_{x}, u_{y}\right)
$$

This equation shows that slippage $\left(s_{x}, s_{y}\right)$ may be separated into three terms. The first term includes two constants, the longitudinal creepage $v_{x}$ and the lateral creepage $v_{y}$. They represent the change in rolling speeds due to the previous mentioned moments $M$ and $M_{x}$. Disregarding temporarily the tangential forces $F_{x}$ and $F_{y}$ developed in the contact region so that there becomes no resistance to $M$ and $M_{x}$, then body 1 's rolling velocity deviates from its original value $\left(V_{0}, 0\right)$. Note that equal but opposite moments $-M$ and $-M_{x}$ are applied to body 2 as well (not shown in Fig. 1), and a change in rolling speed of body 2 also occurs. Let $\left(\delta V_{i x}, \delta V_{i y}\right)$ represents the change in rolling velocity of body $i(i=1$ or 2$)$ from the value $\left(V_{0}, 0\right)$, then longitudinal and lateral creepages $v_{x}$ and $v_{y}$ are defined as

$$
\left(v_{x}, v_{y}\right)=\left(\delta V_{1 x}-\delta V_{2 x}, \delta V_{1 y}-\delta V_{2 y}\right) / V_{0}
$$

The second term of $\left(s_{x}, s_{y}\right)$ is due to the moment $M_{z}$ applied to body 1 , and also the equal but opposite moment $-M_{z}$ applied to body 2 (not shown in Fig. 1). At the point of application of $M_{z}$ on body 1, which we assume to be far away from the contact region, body 1 rotates about the $z$ axis with an angular velocity $\omega_{1}$. Neglecting temporarily the resistant moment $-M_{z}$ on body 1 so that body 1 can rotate freely, then the linear velocity at the point $(x, y)$ in the contact region on body 1 is $\omega_{1}(-y, x)$. The velocity of this point relative to the same point on body 2 is then $\phi(-y, x)$, where the constant $\phi$ is called the relative spin, or simply the spin, defined by the equation 


$$
\phi=\left(\omega_{1}-\omega_{2}\right) / V_{0}
$$

In obtaining the first two slip components, namely $\left(v_{x}, v_{y}\right)$ and $\phi(-y, x)$, the resisting forces $\left(F_{x}, F_{y}\right)$ and resisting moment $M_{z}$ were not taken into account. The elastic deformation caused by these forces (moment) was neglected. Let $u_{x}$ and $u_{y}$ denote the elastic displacements produced by $\left(F_{x}, F_{y}, M_{z}\right)$ at a point $(x, y)$ in the contact region on body 2 . The relative slip velocity due to $\left(u_{x}, u_{y}\right)$ is given by the last term of equation (2).

\section{Discretization}

The elastic displacements $\left(u_{x}, u_{y}\right)$ in Eq. (2) are caused by the resisting forces $F_{x}$, $F_{y}$, and $M_{z}$, which are the resultants of tangential traction $\left(T_{x}, T_{y}\right)$ in the contact region. Using principle of superposition, one may express $\left(u_{x}, u_{y}\right)$ as follows

$$
\left\{\begin{array}{l}
u_{x}(x, y) \\
u_{y}(x, y)
\end{array}\right\}=\iint_{\Omega}\left[\begin{array}{ll}
f_{x x} & f_{x y} \\
f_{y x} & f_{y y}
\end{array}\right]\left\{\begin{array}{l}
T_{x}(\alpha, \beta) \\
T_{y}(\alpha, \beta)
\end{array}\right\} d \alpha d \beta
$$

where $f_{i j}(x, y, \alpha, \beta)$ are Cerruti functions [14]. For example, $f_{x y}$ represents the displacement at the point $(x, y)$, in the $x$ direction, which is produced by a unit force at the point $(\alpha, \beta)$, in the $y$ direction.

The contact region is first replaced by a number of horizontal strips, as Fig. 2(a) shows, and each strip is further divided into several rectangular cells. Hence the contact region is discretized into $n$ rectangular cells. The sub-region of $\Omega$ where relative slip occurs is called the slip zone, denoted by the symbol $\Omega^{S}$. The remaining region of $\Omega$ where relative slip is prohibited is called the stick zone (or locked zone), and we denote it by $\Omega^{L}$. Equation (2) is valid at each cell center, but instead of dealing with this equation directly, Liu and Paul [5], Hsu [15], and also Liu and Hsu [11] integrated Eq. (2) with respect to $x$ to remove the partial derivative. Following the procedure suggested by Liu and Hsu, we integrate both sides of Eq. (2) from the center of the $i^{\prime}$ th cell $\left(x_{i}, y_{i}\right)$, to a point $\left(x_{U}, y_{i}\right)$, also making use of Eq. (5), obtaining

$$
\sum_{j=x i}^{x_{U}} \Delta x_{j}\left\{\begin{array}{l}
s_{x j} \\
s_{y j}
\end{array}\right\}=\left\{\begin{array}{l}
v_{x}\left(x_{U}-x_{i}\right)-\phi y_{i}\left(x_{U}-x_{i}\right) \\
v_{y}\left(x_{U}-x_{i}\right)+\frac{\left(x_{U}^{2}-x_{i}^{2}\right) \phi}{2}
\end{array}\right\}+2 \sum_{j=1}^{n} \iiint_{\Omega_{j}}\left[\begin{array}{ll}
h_{x x} & h_{x y} \\
h_{y x} & h_{y y}
\end{array}\right] d \alpha d \beta\left\{\begin{array}{l}
T_{x j} \\
T_{y j}
\end{array}\right\}
$$

In the last equation $\left(s_{x j}, s_{y j}\right)$ and $\left(T_{x j}, T_{y j}\right)$ are slippage and traction at the center of the $j^{\prime}$ th cell, whose width is $\Delta x_{j}$; functions $h_{i j}$ are defined by

$$
h_{i j}\left(x_{i}, y_{i}, \alpha, \beta\right)=f_{i j}\left(x_{U}, y_{i}, \alpha, \beta\right)-f_{i j}\left(x_{i}, y_{i}, \alpha, \beta\right)
$$

and $\Omega_{j}$ denotes the rectangular region of the $j^{\prime}$ th cell, over which the double integral is evaluated. Tractions and slippages are taken out of the integrals since we assume they take constant values in a cell. The upper limit of integration $\left(x_{U}, y_{i}\right)$ depends upon position of the cell center $\left(x_{i}, y_{i}\right)$. Generally speaking $\left(x_{U}, y_{i}\right)$ is the closest boundary on the right of $\left(x_{i}, y_{i}\right)$. It can either be boundary that separates the slip-stick zone, or boundary of the contact region. For example, if $\left(x_{i}, y_{i}\right)$ is point $A$, point $B$, or point $C$ in Fig. 2(b), then $x_{U}$ is $x_{S 1}, x_{S 2}$, and $x_{E}$, respectively. 


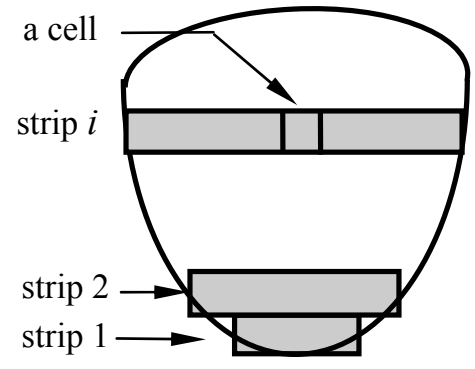

(a)

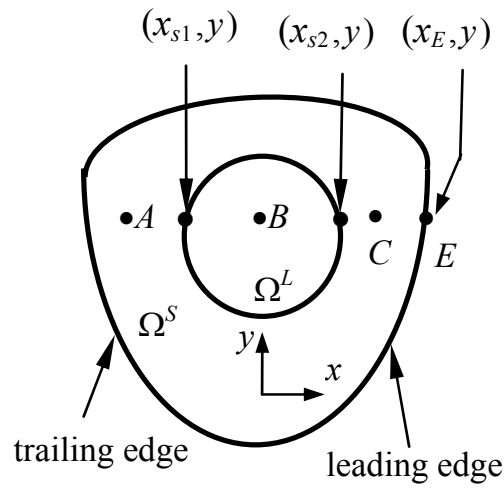

(b)

Figure 2: $\quad$ (a) Discretized contact region; (b) integration limits.

Note that equation (6) can be written at each cell center $\left(x_{i}, y_{i}\right)$, with $i=1,2, \ldots, n$, and for each cell center there are components in the $x$ and in the $y$ directions. Hence equation (6) implies $2 n$ equations. In addition, when $\left(x_{i}, y_{i}\right)$ is in the slip zone, tangential tractions in this cell satisfy Coulomb's law of friction, i.e.

$$
\left(T_{x i}^{S}\right)^{2}+\left(T_{y i}^{S}\right)^{2}-\left(\mu p_{i}\right)^{2}=0, \text { for } i=1,2, \ldots, n^{S}
$$

In the last equation the superscript $S$ means the associated variable is for the slip zone, in particular, $\left(T_{x i}^{S}, T_{y i}^{S}\right)$ is the traction of a cell $i$ in the slip zone, and $n^{S}$ is the number of cells in the slip zone. The traction vector should be collinear with the slip vector, hence

$$
s_{x i} T_{y i}^{S}-s_{y i} T_{x i}^{S}=0, \quad i=1,2, \ldots, n^{S}
$$

Note that the last equation only requires traction and slippage to be collinear. We also require that traction on the surface of body 2 is in exactly the same direction as the relative motion of body 1 to body 2 , hence $\mathbf{s} \cdot \mathbf{T}^{S} \geq 0$, or

$$
s_{x i} T_{x i}^{S}+s_{y i} T_{y i}^{S} \geq 0, \quad i=1,2, \ldots, n^{S}
$$

Therefore we have $2 n+2 n^{S}$ equations, given by Eqs. (6), (8), and (9), for the same number of unknowns, which are the $2 n$ tractions $\left(T_{x i}, T_{y i}\right)$ and the $2 n^{S}$ slippages $\left(s_{x i}, s_{y i}\right)$.

\section{Numerical procedure}

If there are $n^{L}$ cells in the stick zone $\Omega^{L}$, we may write Eq. (6) $n^{L}$ times, each time $\left(x_{i}, y_{i}\right)$ is replaced by a different cell center coordinate in $\Omega^{L}$, then we obtain $2 n^{L}$ equations as follows $[11,15]$

$$
\mathbf{0}=\mathbf{e}-\mathbf{A} \mathbf{t}^{L}-\mathbf{B} \mathbf{t}^{S},\left(x_{i}, y_{i}\right) \in \Omega^{L}
$$

In the last equation $\mathbf{t}^{L}$ and $\mathbf{t}^{S}$ are traction vectors of lengths $2 n^{L}$ and $2 n^{S}$, respectively, representing tractions in the stick and the slip zones; $\mathbf{A}$ and $\mathbf{B}$ are 
coefficient matrices obtained by integrating Cerruti functions over cells in $\Omega^{L}$ and in $\Omega^{S}$, respectively; $\mathbf{e}$ is the vector containing the constants of the first term on the right hand side of Eq. (6). Zero vector at the left hand side is due to the fact that no relative slip occurs in stick cells. Similarly we may write $2 n^{S}$ equations for cells in $\Omega^{S}$, as follows

$$
\mathbf{s}=\mathbf{q}-\mathbf{C t}^{L}-\mathbf{D t}^{S}, \quad\left(x_{i}, y_{i}\right) \in \Omega^{S}
$$

where $\mathbf{s}$ is the slippage vector of length $2 n^{S}$. We may solve $\mathbf{t}^{L}$ from Eq. (11) and then substitute it into Eq. (12), obtaining

$$
\mathbf{s}=\mathbf{q}-\mathbf{C A}^{-1} \mathbf{e}+\left(\mathbf{C A}^{-1} \mathbf{B}-\mathbf{D}\right) \mathbf{t}^{\mathbf{s}}
$$

Thus the procedure is to solve for $\mathbf{s}$ and $\mathbf{t}^{S}$ from equations (8), (9), and (13). After $\mathbf{t}^{S}$ is obtained, then $\mathbf{t}^{L}$ can be found from Eq. (11). Since equations (8) and (9) are nonlinear, the Newton-Raphson scheme is utilized by Liu and Paul [5], Hsu [15], and Liu and Hsu [11]. The initial guesses suggested by Liu and Hsu are given below

Initial guess 1 :

$$
\left(T_{x i}^{S}, T_{y i}^{S}\right)=\frac{\left(v_{x}-\phi y_{i}, v_{y}+\phi x_{i}\right) \mu p\left(x_{i}, y_{i}\right)}{\sqrt{\left(v_{x}-\phi y_{i}\right)^{2}+\left(v_{y}+\phi x_{i}\right)^{2}}},\left(x_{i}, y_{i}\right) \in \Omega_{S}
$$

Initial guess 2:

$$
\left(T_{x i}^{S}, T_{y i}^{S}\right)=\frac{\mu p\left(x_{i}, y_{i}\right)}{\sqrt{\left(x_{i}-x_{P}\right)^{2}-\left(y_{i}-y_{P}\right)^{2}}}\left[-\left(y_{i}-y_{P}\right),\left(x_{i}-x_{P}\right)\right],\left(x_{i}, y_{i}\right) \in \Omega_{S}
$$

where $\left(x_{p}, y_{p}\right)$ is coordinate of a point called spin pole, whose location is to be estimated, and we will discuss this later. These initial guesses will be used in the subsequent analysis.

In addition to the equation-solving procedure discussed in the last paragraph, another iterative procedure is to determine the sizes of the stick and slip zones $[5,11,15]$. This procedure starts by assuming the whole contact region is the stick zone. Tractions of the first iteration $\mathbf{t}^{L}$ can be obtained by solving Eq. (11), but without the last term. In subsequent iterations a slip zone generally appears, then tractions $\mathbf{t}^{S}, \mathbf{t}^{L}$, and slippage $\mathbf{s}$ are obtained using the previous mentioned equation-solving procedure. At the end of the an iteration, if traction at a cell in the stick zone equals to or exceeds its limiting value $\mu p$, then in the next iteration this cell is in the slip zone. Also, if inequality (10) is violated within any cell in the slip zone, then in the next iteration this cell is in the stick zone. The procedure terminates when all tractions in the stick zone fall below their limiting values, and inequality (10) is satisfied throughout the slip zone.

\section{Results and discussions}

In the following analysis the non-Hertzian contact region and the pressure on the region are taken from Paul and Hashemi [12]. By using initial guess 1, the Newton-Raphson procedure may always converge within $1 \%$, provided that a 
coarse mesh with less than 30 cells is used. In a case with very large spin, the stick zone may become very small, and a very fine mesh is necessary to expose this zone. In such a situation initial guess 1 may lead to divergence, and we can only turn to initial guess 2 , which requires an estimation of the spin pole coordinate. We follow a two-stage analysis procedure suggested by Liu and Hsu [11] for Hertzian contact. In the first stage initial guess 1 with a coarse mesh is used, tractions so obtained may indicate the spin pole location. With this spin pole location, in the second analysis one may use initial guess 2 in a fine mesh. We found this procedure also work for non-Hertzian contact.

Figure 3 shows slip-stick boundaries for various values of normalized spin $\Phi$, defined by $\Phi=E a^{3} \phi /\left[\mu N\left(1-\sigma^{2}\right)\right]$, when the normalized longitudinal creepage $E a^{2} v_{x} /\left[\mu N\left(1-\sigma^{2}\right)\right]=0.6 ; E$ is modulus of elasticity, $\sigma$ is Poisson's ratio, and $a$ is the half length of the longest strip in the contact region, as shown in the figure. Figure 4 shows both the slip and stick zones under pure spin. One may notice that the stick zone may reduce to separated poles. In figure 5 we show the resultant force $F_{x}$ and moment $M_{z}$ due to longitudinal creepage $v_{x}$. All these results show that the initial guesses 1 and 2 may give convergent results under non-Hertzian contact.

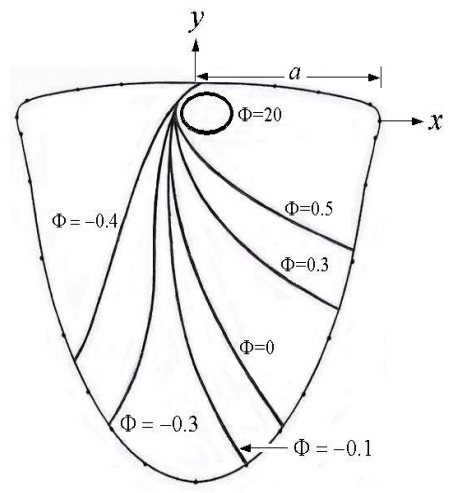

Figure 3: Slip-stick boundaries under various spin when $E a^{2} v_{x} /\left[\mu N\left(1-\sigma^{2}\right)\right]=0.6, \Phi=E a^{3} \phi /\left[\mu N\left(1-\sigma^{2}\right)\right]$.

\section{Conclusions}

In this study it is shown that the numerical technique suggested by Liu and Hsu for Hertzian contact may be extended to cases with non-Hertzian contact. The two initial guesses suggested by them may be used together and convergence may always be obtained. For cases with large spins, initial guess 1 under a coarse mesh may provide spin pole locations, which are used in the analysis using initial guess 2, with a much finer mesh. 


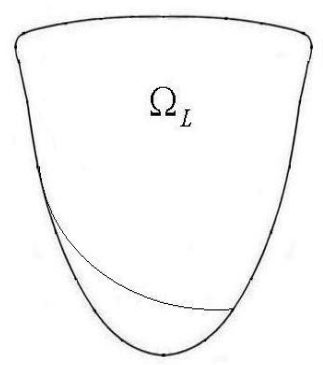

(a) $\Phi=0.37$

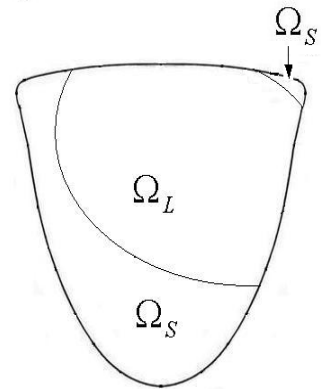

(c) $\Phi=1.04$

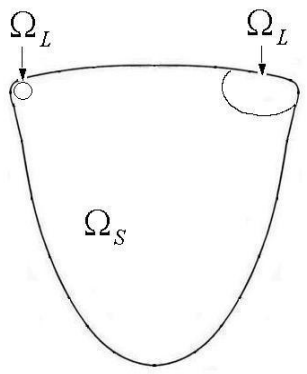

(e) $\Phi=1.63$

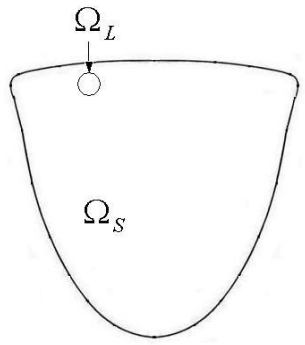

(g) $\Phi=5.19$

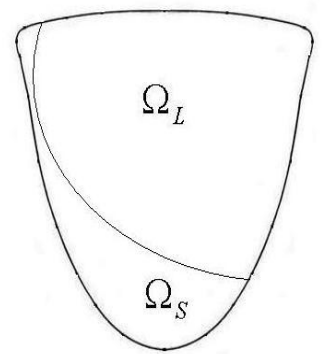

(b) $\Phi=0.74$

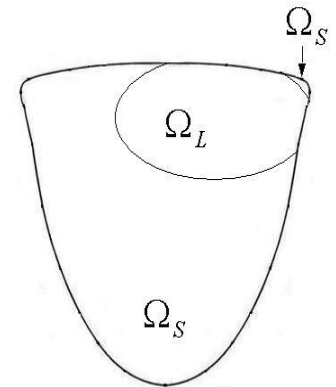

(d) $\Phi=1.34$

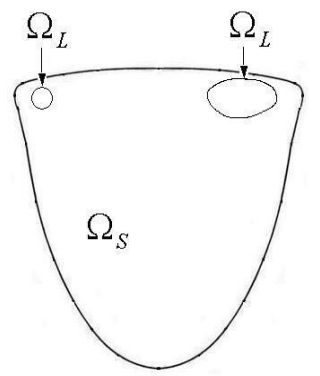

(f) $\Phi=1.78$

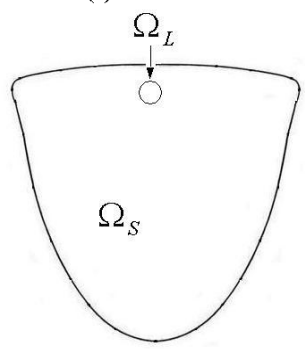

(h) $\Phi=22.3$

Figure 4: $\quad$ Slip and Stick zones under pure spin. 


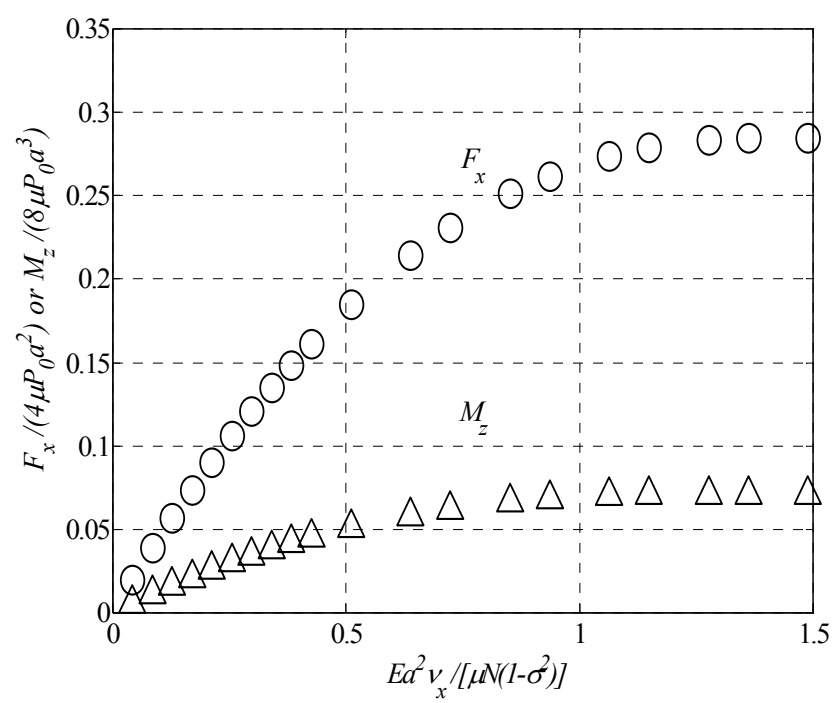

Figure 5: Tangential force $F_{x}$ and twisting moment $M_{z}$ due to longitudinal creepage $v_{x}$.

\section{Acknowledgement}

The authors gratefully acknowledge that this study was partially supported by the National Science Council of ROC under grant no. NSC95-2212-E-032-015.

\section{References}

[1] Johnson, K.L., The Effect of a Tangential Contact Force upon the Rolling Motion of an Elastic Sphere on a Plane, Journal of Applied Mechanics, 25, pp. 339-346, 1958.

[2] Johnson, K.L., The Effect of Spin upon the Rolling Motion of an Elastic Sphere on a Plane, Journal of Applied Mechanics, 25, pp. 332-338, 1958.

[3] Carter, F.W., On the Action of a Locomotive Driving Wheel, Proceedings of Royal Society, Vol. A112, pp. 151-157, 1926.

[4] Hills, D.A, Nowell, D., and Sackfield, A., Mechanics of Elastic Contact, Butterworth-Heinemann, 1993.

[5] Liu, C. and Paul, B., Rolling Contact With Friction and Non-Hertzian Pressure Distribution, Journal of Applied Mechanics, 56(4), pp. 814-820, 1989.

[6] Zastrau, B., Nackenhorst, U., \& Jarewsky, J., On the Computation of Elastic-Elastic Rolling Contact Using Adaptive Finite Element Techniques, Proceeding of $3^{\text {rd }}$ International Conference on Contact Mechanics, pp. 129-138, 1997.

[7] Wriggers, P., Computational Contact Mechanics, $2^{\text {nd }}$ ed., Springer, 2006. 
[8] Kalker, J.J., On the Rolling Contact of Two Elastic Bodies in the Presence of Dry Friction, Ph. D. dissertation, Delft University of Technology, Delft, Netherlands, 1967.

[9] Kalker, J.J., Computation of Three-Dimensional Rolling Contact With Dry Friction, International Journal for Numerical Methods in Engineering, 14(9), pp. 1293-1307, 1979.

[10] Kalker, J.J., Wheel-rail wear calculations with the program CONTACT, Contact Mechanics and Wear of Rail-Wheel Systems II, University of Waterloo Press, Waterloo, Ontario, pp.3-26, 1987.

[11] Liu, C.H. and Hsu, W-E, "Three-Dimensional Rolling Contact Stress Analysis", in Computer Methods and Experimental Measurements for Surface Effects and Contact Mechanics VII, editors J. T. M. de Hosson, C. A. Brebbia, and S-I Nishida. WIT press, pp. 269-278, 2005.

[12] Paul, B. and Hashemi, J. "User's Manual for Program COUNTACT (COUNTerformal contACT stress programs)," Technical Report No. 4, FRA-ORD-78-72, (PB 286097/AS, available from National Technical Information Service, Springfield, Va.22151), 1977.

[13] Johnson, K.L., Contact Mechanics, Cambridge University Press, 1985.

[14] Love, A.E.H., A Treatise On The Mathematical Theory Of Elasticity, New York: Dover Publications, 1944.

[15] Hsu, W-E., Three-dimensional Rolling Contact Stress Analyses, Master thesis, Dept. of mechanical \& electro-mechanical engineering, Tamkang University, 2005, in Chinese. 